\title{
Orbital cellulitis in West Texas
}

\author{
Cameron Clarke MD, Kelly Mitchell MD
}

\begin{abstract}
Purpose: Identify the risk factors, microbiology, complications, and treatment outcomes of orbital cellulitis in West Texas.

Study type: Retrospective chart review.

Main findings: Forty-six patients over a 10-year period were treated for orbital cellulitis. The majority of patients were adult and male. The most common causative organisms were Staphylococcus aureus and polymicrobial infections. Abscess formation, either subperiosteal or intraorbital, was the most common complications. The majority of patients regained normal visual acuity following resolution of the infection.

Conclusion: Prompt treatment of orbital cellulitis results in limited complications and resolution of visual loss.
\end{abstract}

Keywords: orbital cellulitis, subperiosteal abscess, preseptal cellulitis

\section{INTRODUCTION}

Orbital cellulitis is one of the few ophthalmic emergencies in which delays in treatment can result in the loss of vision, loss of an eye, or even loss of life. Infections of the orbit are defined by their anatomical location relative to a membranous extension of periosteum in the eyelid called the orbital septum. Orbital cellulitis occurs posterior to the septum (post-septum) and involves the soft tissue of the orbit (extraocular muscles, optic nerve, eye); pre-septal infections affect only the superficial soft tissues of the eyelid and surrounding facial tissue. Orbital cellulitis is rare, affecting 1.6-3.5 per 100000 people each year, but is ten times more common in pediatric patients than adults. ${ }^{1}$ Given its propensity for serious complications, including intracranial abscess, cavernous sinus thrombosis, and meningitis, it is important for clinicians to recognize the signs and symptoms of orbital cellulitis and initiate prompt treatment to halt the natural progression

Corresponding author: Kelly Mitchell Contact Information: Kelly.mitchell@ttuhsc.edu DOI: 10.12746/swrccc.v9i40.829 of disease. ${ }^{2}$ Signs suggesting post-septal infection include decreased vision, restriction of extraocular motility, and conjunctival swelling or injection.

Predisposing factors for orbital cellulitis include orbital trauma, preseptal cellulitis, dental caries, and sinusitis. ${ }^{3}$ Streptococcus pneumoniae, Staphylococcus aureus, Haemophilus influenzae, and anaerobic bacteria are the most commonly reported micro-organisms in the literature. ${ }^{4}$ However, the majority of cases are culture negative, necessitating treatment with empiric antibiotics. ${ }^{5}$ While medical management alone is often sufficient, surgical intervention may be required for abscess formation. ${ }^{6}$

The purpose of this study is to update the epidemiology, risk factors, microbiological composition, major complications, and treatment outcomes of orbital cellulitis at a tertiary care referral center in West Texas.

\section{Methods}

A retrospective chart review of all cases of orbital cellulitis treated by the Department of Ophthalmology from January 2010 to May 2020 was conducted with the approval of the Institutional Review Board. 
Table 1. Demographics and Risk Factors for Orbital Cellulitis

\begin{tabular}{|c|c|c|c|c|c|c|}
\hline & \multicolumn{2}{|c|}{ All $(n, \%)$} & \multicolumn{2}{|c|}{ Children (n, \%) } & \multicolumn{2}{|c|}{ Adult (n, \%) } \\
\hline Total patients & 46 & 100.0 & 20 & 43.5 & 26 & 56.5 \\
\hline Male & 29 & 63.0 & 13 & 65.0 & 16 & 61.5 \\
\hline Female & 17 & 37.0 & 7 & 35.0 & 10 & 38.5 \\
\hline \multicolumn{7}{|l|}{ Race } \\
\hline White & 20 & 43.5 & 7 & 35.0 & 13 & 50.0 \\
\hline Hispanic & 19 & 41.3 & 8 & 40.0 & 10 & 38.5 \\
\hline Black & 6 & 13.0 & 4 & 20.0 & 2 & 7.7 \\
\hline Asian & 1 & 2.2 & 1 & 5.0 & 0 & 0.0 \\
\hline \multicolumn{7}{|l|}{ History } \\
\hline Sinusitis & 33 & 71.7 & 17 & 85.0 & 16 & 61.5 \\
\hline Preseptal cellulitis & 14 & 30.4 & 6 & 30.0 & 8 & 30.8 \\
\hline Trauma & 10 & 21.7 & 5 & 25.0 & 5 & 19.2 \\
\hline Dental caries & 5 & 10.9 & 1 & 5.0 & 4 & 15.4 \\
\hline Recent facial surgery & 3 & 6.5 & 0 & 0.0 & 3 & 11.5 \\
\hline Foreign body & 1 & 2.2 & 0 & 0.0 & 1 & 3.8 \\
\hline
\end{tabular}

Inclusion criteria included all cases of orbital cellulitis confirmed by either computed tomography (CT) or magnetic resonance imaging (MRI) studies. Cases diagnosed clinically without corroborating radiological exams and those with incomplete charts were excluded from analysis.

Of the initial 87 patients initially returned in chart review, $41(47.1 \%)$ were excluded from analysis. Twenty-four $(58.5 \%)$ of those excluded were found to have pre-septal cellulitis when their radiological studies were reviewed. The remaining 46 (52.9\%) patients were selected for further analysis. Data collected included patient demographics, medical history, current medications, risk factors, ophthalmological examination, CT/MRI imaging results, surgical interventions, microbiology results, complications, and examination findings from follow-up appointments. Primary outcomes were post-recovery visual acuity and major complications associated with orbital cellulitis. Secondary outcomes included the etiology of the infection, duration of antibiotic therapy, and duration of hospitalization.

Data analysis was conducted using Microsoft Excel (Redmond, WA).

\section{Results}

Forty-six patients with radiologically confirmed orbital cellulitis were identified in the study period. The majority of patients were adults $(26,56.5 \%)$ and male $(29,63.0 \%)$ (Table 1$)$. In adults, the median age was 44; in children, it was nine. The overall age range was $0-85$. There were more males affected in all age groups. There were similar numbers of Caucasian $(20,43.5 \%)$ and Hispanic $(19,41.3 \%)$ patients. Most patients had a normal body mass index $(28,60.9 \%)$; $17(37.0 \%)$ were clinically obese.

Sinusitis was the predominant etiology identified in $33(71.7 \%)$ cases, but some patients had multiple predisposing factors contributing to development of post-septal cellulitis. Fourteen patients $(30.4 \%)$ had pre-septal cellulitis prior to development of orbital symptoms, and ten patients $(21.7 \%)$ had a traumatic injury to the orbit. Only five patients $(10.9 \%)$ had type 2 diabetes mellitus, and only one patient $(2.2 \%)$ had HIV. Two patients (4.4\%) developed sepsis during their hospital admission.

The most common complications of orbital cellulitis during hospitalization were intraorbital $(11,23.9 \%)$ or 


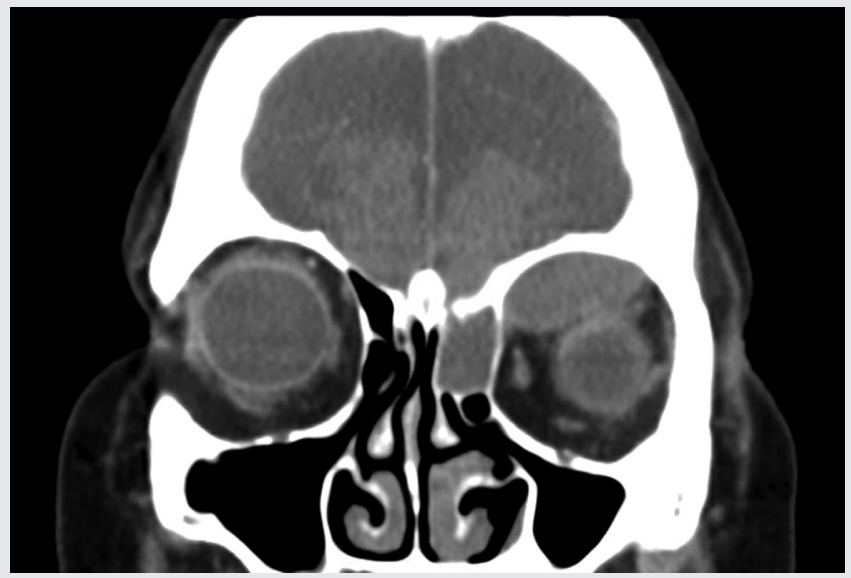

Figure 1. An axial CT image of a large subperiosteal abscess of the right nasal orbit.

subperiosteal abscess (19, 41.3\%) (Figure 1), exposure keratopathy $(6,13.0 \%)$, and meningitis $(4,8.7 \%)$ (Table 2). Subperiosteal abscess was much more common in pediatric patients; 12 (60\%) developed this complication compared to seven adults $(26.9 \%)$.

The average length of stay was 4.4 days for pediatric patients and 7.9 days for adults. Antibiotics were used intravenously for a median of 4.5 days and then orally for 10 days after discharge. Thirty-five patients (75\%) received broad-spectrum antibiotic coverage with vancomycin plus a penicillin agent, such as piperacillin/ tazobactam or ampicillin/sulbactam. Clindamycin or amoxicillin/clavulanic acid were the most commonly
Table 2. Complications of Orbital Cellulitis

\begin{tabular}{|l|r|r|}
\hline Complications & \multicolumn{2}{|c|}{ All (n, \%) } \\
\hline Subperiosteal abscess & 19 & 41.3 \\
\hline Elevated intraocular pressure & 12 & 26.1 \\
\hline Intraorbital abscess & 11 & 23.9 \\
\hline Exposure keratopathy & 6 & 13.0 \\
\hline Meningitis & 4 & 8.7 \\
\hline Cavernous sinus thrombosis & 2 & 4.3 \\
\hline Reoperation & 2 & 4.3 \\
\hline Enucleation & 1 & 2.2 \\
\hline Endophthalmitis & 0 & 0.0 \\
\hline
\end{tabular}

prescribed oral antibiotics for both groups. Extended intravenous antibiotic courses were given to four adults $(15 \%)$ and one pediatric patient $(5 \%)$. Almost half of all patients $(21,46.5 \%)$ required a surgical intervention for abscess drainage or exploration. Eleven of the surgical cases were transcutaneous irrigation and drainage by ophthalmology. The remaining ten underwent endoscopic sinus irrigation and drainage through the otolaryngology service.

Half of all patients (23) had a positive microbiological culture obtained (Table 3 ). The most common bacteria identified were methicillin-resistant $S$. aureus $(6$, $26.1 \%)$, polymicrobial infections $(21.7 \%)$, methicillinsusceptible $S$. aureus $(4,17.4 \%)$, and viridans streptococci $(2,8.7 \%)$. Polymicrobial infections included

Table 3. Microbiology of Identified Organisms

\begin{tabular}{|l|c|c|c|c|c|c|}
\hline Microbiology & \multicolumn{2}{|l|}{ All (n, \%) } & \multicolumn{2}{c|}{ Pediatrics (n, \%) } & \multicolumn{2}{c|}{ Adult (n, \%) } \\
\hline Positive cultures & 23 & 50.0 & 9 & 45.0 & 14 & 53.8 \\
\hline MRSA & 6 & 26.1 & 2 & 22.2 & 5 & 35.7 \\
\hline Polymicrobial & 5 & 21.7 & 3 & 33.3 & 2 & 14.3 \\
\hline MSSA & 4 & 17.4 & 1 & 11.1 & 2 & 14.3 \\
\hline Streptococcus viridans & 2 & 8.7 & 1 & 11.1 & 1 & 7.1 \\
\hline Staph. epidermidis & 2 & 8.7 & 1 & 11.1 & 1 & 7.1 \\
\hline Group A Strep. & 1 & 4.3 & 1 & 11.1 & 0 & 0.0 \\
\hline Rhizopus sp. & 1 & 4.3 & 0 & 0.0 & 1 & 7.1 \\
\hline Klebsiella aerogenes & 1 & 4.3 & 0 & 0.0 & 1 & 7.1 \\
\hline Aeromonas hydrophila & 1 & 4.3 & 0 & 0.0 & 1 & 7.1 \\
\hline
\end{tabular}


common Gram negative and positive oral flora. There were no significant differences between causative species among adults, children, and patients with subperiosteal abscesses.

The initial vision on presentation was on average 20/100 (logMAR: 0.72), and there were 23 cases of orbital cellulitis in each eye. One patient developed orbital cellulitis in her fellow orbit 14 days following discharge for her initial episode with pansinusitis and subperiosteal abscess. Eleven patients $(23.9 \%)$ required treatment for elevated intraocular pressure. Only $57 \%$ of patients presented for scheduled follow-up in clinic after hospital discharge. Of those returning for follow-up, average visual acuity was 20/35 (logMAR: 0.244 ).

\section{Discussion}

As the tertiary referral center for West Texas and Eastern New Mexico, University Medical Center in Lubbock, Texas, serves a diverse patient population with many medical comorbidities and complicated presentations of disease processes. However, orbital cellulitis occurred more commonly in young, healthy individuals without major medical comorbidities. Most cases resolved without surgical management, and infections were almost exclusively from Gram positive bacteria. The most common complications were subperiosteal and intraorbital abscess formation and elevated intraocular pressure. In this study, patients without serious complications had complete vision recovery.

Orbital cellulitis was more common in adults (56.5\%) than in children (43.5\%). This is in contrast to other studies in Scotland, ${ }^{1}$ New York, $^{2}$ and the Philippines ${ }^{7}$ that reported a preponderance of cases in younger populations. This may be due to the referral base transferring to our hospital for a higher level of care with pediatric patients being either treated in their home institutions or transferred to other hospitals. There were no significant differences in etiology between adults and children. The most common predisposing factors leading to development of post-septal cellulitis were sinusitis $(71.7 \%)$, pre-septal cellulitis $(30.4 \%)$, and trauma $(21.7 \%)$, consistent with other major studies in the literature. The former two etiologies identify a feasible area for prevention of orbital cellulitis: early diagnosis and treatment by primary care physicians allows for optimal outpatient treatment and may prevent progression to the vision and life-threatening complications associated with orbital cellulitis. ${ }^{2}$

The most common bacterial organisms isolated in the literature are Staphylococcal sp, Streptococcal sp, and Haemophilus sp. ${ }^{4,8}$ Our results are similar, and MRSA (26\%) was the most common etiology. There were no significant differences in bacterial species between adult and pediatric patients. Most patients in the current study received intravenous antibiotics during their initial hospitalization with extended oral antibiotic regimens following discharge. We found a significantly lower rate of vancomycin usage in pediatric patients $(40 \%)$ than in previously published literature. ${ }^{9}$ This may be due to provider preference and resistance rates in our area to common antibiotics, such as clindamycin. Although intravenous antibiotics are often used, a prospective study by Cannon et al. found no difference in hospitalization duration or complications when using highly bioavailable oral antibiotics. ${ }^{5}$ The authors highlight oral antibiotics allow for rapid initial dosing, easier therapy in children, and decreased interruption in treatment. Regardless, we recommend initial broad-spectrum antibiotic coverage, such as vancomycin combined with a penicillin and beta lactamase inhibitor combination drug, followed by a course of oral antibiotics with excellent soft tissue penetration such as clindamycin or amoxicillin/clavulanic acid.

Broad-spectrum antibiotic therapy decreases the rate of serious complications from orbital cellulitis. ${ }^{1}$ Our study included 6 cases with infections extending posterior to the orbit causing meningitis or cavernous sinus thrombosis. All of these patients had a full recovery. The one case of enucleation was in a patient who previously had undergone evisceration (removal of intraocular contents while closing the scleral shell).

Subperiosteal abscess formation was the most frequent complication requiring surgical intervention in nearly two-thirds of our cases. These abscesses have been associated with younger age, restricted extraocular movement, and prior antibiotic use. ${ }^{3}$ Garcia and Harris recommended surgical drainage of subperiosteal abscesses for patients with frontal sinusitis, acute optic nerve compromise, and older than nine years of age. ${ }^{6}$ 
Additionally, abscesses larger than $2 \mathrm{~cm}$ benefit from combined sinus and abscess drainage. ${ }^{2}$ We found no difference in post-hospitalization visual acuity between patients with and without a subperiosteal abscess.

Our study is limited by its retrospective design and limited patient follow-up after discharge. Inadequate follow-up may be related to low medical literacy, unsatisfactory outcomes, and/or prohibitive costs associated with travel, appointments, or medication costs.

Orbital cellulitis can be a rapidly evolving complex medical emergency, and optimal care requires a coordinated interdisciplinary team involving multiple specialties. Optimal treatment focuses on prompt initial assessment including computed tomographic scanning of the orbit and maxillofacial region, admission with broad-spectrum intravenous antibiotics, close follow-up with multiple serial exams during the initial 24 hours, and surgical intervention for drainage of concerning subperiosteal abscesses, if needed. The results of this study can help guide therapeutic intervention for cases of orbital cellulitis on the South Plains.

Article citation: Clarke C, Mitchell K. Orbital cellulitis in West Texas. The Southwest Respiratory and Critical Care Chronicles 2021;9(40):9-13

From: Department of Ophthalmology, Texas Tech University Health Sciences Center, Lubbock, Texas

Submitted: $3 / 31 / 2021$

Accepted: 5/1/2021

Reviewer: Jacob Nichols MD

Conflicts of interest: none

This work is licensed under a Creative Commons

Attribution-ShareAlike 4.0 International License.

\section{REFERENCES}

1. Murphy C, Livingstone I, Foot B, et al. Orbital cellulitis in Scotland: Current incidence, aetiology, management and outcomes. Br J Ophthalmol 2014;98(11):1575-1578.

2. Dewan MA, Meyer DR, Wladis EJ. Orbital cellulitis with subperiosteal abscess: Demographics and management outcomes. Ophthal Plast Reconstr Surg 2011;27(5):330-332.

3. Erickson BP, Lee WW. Orbital Cellulitis and Subperiosteal Abscess: A 5-year Outcomes Analysis. Orbit 2015;34(3): 115-120.

4. Ferguson MP, McNab AA. Current treatment and outcome in orbital cellulitis. Aust N Z J Ophthalmol. 1999;27(6):375-379.

5. Cannon PS, Keag DM, Radford R, et al. Our experience using primary oral antibiotics in the management of orbital cellulitis in a tertiary referral centre. Eye 2009;23(3):612-615.

6. Garcia GH, Harris GJ. Criteria for nonsurgical management of subperiosteal abscess of the orbit: analysis of outcomes 1988-1998. Ophthalmology 2000;107(8):1454-1456; discussion 1457-8.

7. Uy HS, Tua o PMC. Preseptal and orbital cellulitis in a developing country. Orbit 2007;26(1):33-37.

8. Atfeh MS, Khalil HS. Orbital infections: Five-year case series, literature review and guideline development. J Laryngol Otol 2015;129(7):670-676.

9. Seltz LB, Smith J, Durairaj VD, et al. Microbiology and antibiotic management of orbital cellulitis. Pediatrics. 2011;127(3): e566-e572. 